\title{
Assessment of Air Way Resistance Indexes and Exercise-Induced Asthma after a Single Session of Submaximal Incremental Aerobic Exercise
}

\author{
by \\ Rouholah Fatemi ${ }^{1}$, Mohsen Ghanbarzadeh ${ }^{1}$
}

\begin{abstract}
The present study aimed at assessing air way resistance indexes that include FEV1 (Force expiration Volume in one second), FVC (Forced vital capacity) and FEV1/FVC and exercise-induced asthma (EIA) after one session of sub maximal incremental aerobic exercise. Fifty healthy male subjects (age 19-26) from the faculty of Physical Education, University of Shahid Chamran served as the participants of the study. They were randomly assigned to either exercise or control groups. Body height, body mass and pulmonary factors were measured in the pre-test conditions. The study protocol included a sub maximal incremental Astrand - Rhyming test on an ergocycle. After performing this test by the exercise group, FEV1, FVC and FEV1/FVC, were measured again for both groups and compared with pre test evaluations. The data were analyzed through descriptive and inferential statistics (dependent and independent $t$ test). Results showed that there was a significant difference in FEV1 between the two groups after the exercise protocol $(p \leq 0.05)$. There was no significant difference in FVC between the two groups after exercise, and a significant difference was registered in FEV1 and FEV1/FVC between pre-test and post-test results in the group that performed the aerobic test protocol $(p \leq 0.05)$. Our results indicate that one sub maximal incremental aerobic exercise session causes a significant change in FEV1 and FEV1/FVC, and causes exercise-induced asthma.
\end{abstract}

Key words: airway resistance, submaximal test, FEV1, FVC

\section{Introduction}

Diurnal variations may lead to changes in athlete's performance through changing blood, metabolic and pulmonary responses at rest and during exercise. As concluded by the Buffalo health institute, pulmonary function is a long term predictor of over all survival rates, in both the genders and it could be used as a tool in general health assessment (Holger, 2000, Prakash, 2007).

Pulmonary ventilation also has a linear relationship with oxygen uptake at different levels of exercise. According to Fatemi (2009), pulmonary function parameters have close interactions with maximum oxygen uptake $\left(\mathrm{VO}_{2 \max }\right)$, and subjects with higher
$\mathrm{VO}_{2 \max }$ have a lower airway resistance. Also, the effect of exercise on pulmonary muscles has been verified in athletes, as they pose higher lung capacity in comparison to untrained subjects (Prajapati, 2008).

Besides, more studies have revealed that exercise can result in broncospasm and can lead to an increase in airway resistance which is referred to as Exercise-Induced Asthma (EIA) (Ucok, 2004, Smith, 2002, Mehmet, 2004). Among methods for EIA diagnosis, there are several incremental exercise protocols. In such methods, the respiratory assessment of FEV1/FVC is evaluated after each stage of the protocol. The measurements are taken in 20-minute intervals. The diagnostic standard for EIA was considered as a 15\% decline in FEV1 (Fatemi, 2009, Tan, 1998), more than $10 \%$ decline in FEV1 or $15 \%$ in force

\footnotetext{
${ }^{I}$ - Department of Sport Physiology, School of Physical education\& Sport Science, Shahid Chamran University of Ahvaz, Iran
} 
expiratory flow $25-75 \%$ (FEF 25-75\%) or more than $25 \%$ in Peak expiratory flow rate (PEFR) (Rundell, 1999).

Exercise-induced bronchospasm (EIB) leads to an increase in airway resistance and ventilation-perfusion ratio (VA/Q) in the lungs; and may lead to a drop in partial pressure of arterial oxygen content (PaO2) (Rundell, 1999). FEV1 is a valuable factor for the assessment of airflow obstruction if defined as a ratio of whole expiratory air. The normal rate of this ratio is $75-85 \%$ of FVC volume. In healthy individuals, FEV1 is more than $80 \%$ of FVC (Guyton, 2006).

Exercise is one of the most common reasons for the aggression of asthma, yet sometimes it causes only mild symptoms (Ehteshami, 2002). Other studies also have shown that EIB occurs in $90 \%$ of asthmatic subjects and $40 \%$ of subjects with airway sensitivity (Provost-Craig, 1996, Katz, 1992). Furthermore, expiratory limitations during exercise with moderate and high intensity in elite male and female athletes has been reported in which three potential factors such as diffusion capacity, ventilatory muscle power and limitation in expiration flow have been recognized to limit aerobic capacity (Rundell, 1999).

Nischome et al (2005), surveyed EIB change with perturbation device that decreased resistance against airflow in normal subjects. They showed that the application of this device results in the declination of airway resistance. When subjects breathe through a resistant duct, they cannot achieve optimal levels of $\mathrm{O}_{2}$ and this condition leads to a decrease in peak performance of athletes (Feinstein, 1996, Oguz, 2002).

Some studies have shown that the highest rate of bronchospasm occurs following high intensity exercise which is performed in cold environments (Smith, 2002, Rundell, 1999, Alex, 2006, Jonathan, 2008, Ozturan, 1999). Medelli et al (2006) after measuring FEV1, FEV1/FVC and FEF25-75\% of elite cyclists, found a $52 \%$ obstruction of upper airways in these athletes.

Likewise, it has been shown that the effect of one endurance exercise session with high intensity can result in exercise-induced arterial hypoxemia, respiratory resistance, and inadequate compensatory hyperventilation (Thomas, 2001, Dempsey, 1999).

Considering the varied data in this issue, the authors attempted to assess airway resistance indexes that included FEV1, FVC, FEV1/FVC and exerciseinduced asthma after one session of submaximal incremental aerobic exercise.

\section{Materials and Methods}

\section{Subjects}

Subjects that participated in this study were untrained men that studied at Shahid Chamran University of Ahvaz in Iran. The hundred twenty subjects that volunteered for the study were young (age 19-24), healthy nonsmoking students. Before the administration of the tests, subjects completed health and physical activity level forms. Afterwards, we selected 50 subjects whose scores were in the same range $(\mathrm{n}=50)$; had a mean age of $22.1 \pm 2.4$ years, body height of $174.4 \pm 6.3 \mathrm{~cm}$, body mass of $72.4 \pm 9.7$ $\mathrm{kg}$ and a mean $\mathrm{VO}_{2}$ max of $38.8 \pm 9.8$ ml.kg.min-1. The subjects were randomly assigned to control and exercise groups. All subjects completed an informed consent for participation in the study.

\section{Preliminary procedures}

Tests were performed in the laboratory which was maintained at $24-26{ }^{\circ} \mathrm{C}$ between 10 and $12 \mathrm{am}$. All the participants had breakfast at $7 \mathrm{am}$. They had no history of any major diseases and were not under physical training and did not take any medications. All were informed about the purpose, requirements and the experimental protocol of the investigation. Experimental procedures were demonstrated to allay their apprehension. Body height and body mass of the subjects were measured with the help of height measuring stand and a medical scale (Krups, New Delhi, India). Body mass was measured to an accuracy of $\pm 0.25 \mathrm{~kg}$ and height to an accuracy of \pm $0.5 \mathrm{~cm}$.

\section{Lung Function Testing}

Airway resistance indexes (FEV1, FVC and FEV1/FVC) were measured by a digital spirometer (HI-601, Japan). To measure FVC, each subject blew into the instrument with maximum force after full inspiration; three readings were taken and the best was recorded. Spirometry included FEV1, FVC and FEV1/FVC. The tests were conducted before and at the end of the Astrand - Rhyming test.

\section{Exercise Protocol}

A standard exercise protocol for this study included a submaximal Astrand-Rhyming test performed on a Monark ergocycle. The duration of this test, based on heart rate, was set for 6 minutes, yet it 
Table 1

Subject characteristics and pulmonary function values

\begin{tabular}{ll}
\hline Variables & mean \pm SD \\
\hline Age, $($ yrs $)$ & $22.1 \pm 2.47$ \\
Body mass $(\mathrm{kg})$ & $72.48 \pm 9.72$ \\
Body height $(\mathrm{cm})$ & $174.44 \pm 6.38$ \\
FEV1 (lit) & $3.41 \pm .66$ \\
FEV1, \% predicted & $87.66 \pm 8.78$ \\
FVC (lit) & $3.96 \pm .56$ \\
FVC, \% predicted & $75.28 \pm 8.33$ \\
FEV1/FVC (lit) & $.84 \pm 5.91$ \\
Vo2max, (ml.kg-1..min- $\left.{ }^{1}\right)$ & $38.83 \pm 9.80$ \\
\hline
\end{tabular}

FVC=Forced vital capacity; FEV1=Forced expiratory volume in one second; $V_{2 \max }=$ Maximal oxygen uptake

Table 2

Comparison between pulmonary variables under pre andpost exercise conditions of the control group

\begin{tabular}{ccc}
\hline Variables & Pre test & Post test \\
\hline FEV1 (lit) & $4.24 \pm 0.53$ & $4.19 \pm 0.57^{*}$ \\
FVC (lit) & $4.41 \pm 0.39$ & $4.43 \pm 0.47$ \\
FEV1/FVC (lit) & $0.94 \pm 0.45$ & $0.96 \pm 0.51$ \\
\hline
\end{tabular}

Table 3

Comparison of pulmonary variables under pre and post test conditions of exercise group

\begin{tabular}{ccc}
\hline Variables & Pre test & Post test \\
\hline FEV1 (lit) & $4.25 \pm 0.48$ & $3.50 \pm 0.56^{*}$ \\
FVC (lit) & $4.49 \pm 0.42$ & $4.40 \pm 0.53$ \\
FEV1/FVC (lit) & $0.94 \pm 0.42$ & $0.79 \pm 0.57$ \\
\hline
\end{tabular}

Table 4

Comparison of pulmonary variables under pre and

\begin{tabular}{ccccc}
\hline \multirow{2}{*}{ Variables } & \multicolumn{2}{c}{ Control group } & \multicolumn{2}{c}{ Exercise group } \\
& Pre test & Post test & Pre test & Post test \\
\hline FEV1lit) & $4.24 \pm 0.53$ & $4.19 \pm 0.57$ & $4.25 \pm 0.48$ & $3.50 \pm 0.56^{*}$ \\
FVC (lit) & $4.41 \pm 0.39$ & $4.43 \pm 0.47$ & $4.49 \pm 0.42$ & $4.40 \pm 0.53$ \\
FEV1/ & $0.94 \pm 0.45$ & $0.96 \pm 0.51$ & $0.94 \pm 0.42$ & $0.79 \pm 0.57^{*}$ \\
FVC (lit) & \multirow{5}{*}{$-p<0,05$} \\
\hline \multicolumn{5}{c}{} \\
\hline
\end{tabular}

could have been extended. The initial load was 98 watts. If the heart rate during the first 2 minutes of the test was less than $60 \%$ of HRmax, the load was increased by $49 \mathrm{~W}$ per 2 minutes; whereas if heart rate was in the $60-70 \%$ range of HRmax, the load was increased by $24.5 \mathrm{~W}$ during the 2 minute intervals. If heart rate rose above $70 \%$ of HRmax, the test was continued without a load change until the heart rate reached a steady state and test was terminated.

\section{Statistical Analyses}

All results were presented as mean \pm SD. The spirometric data was expressed in liters. Statistical analysis was performed using the t-test. Values of $\mathrm{p}<0.05$ were considered significant. Statistical analyses were performed using the 17 release version of SPSS for Windows.

\section{Results}

Table 1 summarizes the characteristics of the studied subjects. Comparison between pre test and post test values of FEV1, FVC and FEV1/FVC of the control group are presented in Table 2. No significant differences were observed between these variables under pre and post test conditions (Table 2, Fig.1). Table 3 shows a comparison of pre and post test results of FEV1, FVC and FEV1/FVC of the exercise group. Absolute values of FEV1 and FEV1/FVC decreased after the test in the exercise group, but there was not significant different in FVC after the test protocol (Table 3, Fig.1). Significant differences in FEV1 and FEV1/FVC were also observed between the two groups after the test (Fig. 2, Table 4). These results show that the decrease in FEV1 and FEV1/FVC is $18 \%$ and $15 \%$ respectively. According to the literature, Exercise - induced asthma occurred in the exercise group in the present study (Alex, 2006, Fatemi, 2002).

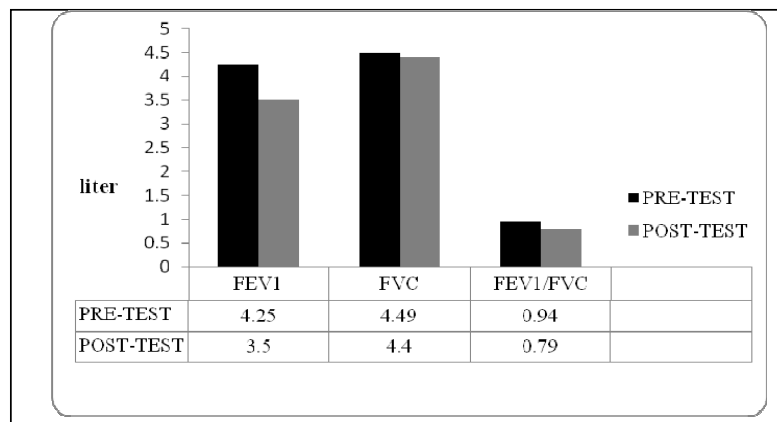

Figure 1

Changes of pulmonary indexes after the test protocol in the exercise group

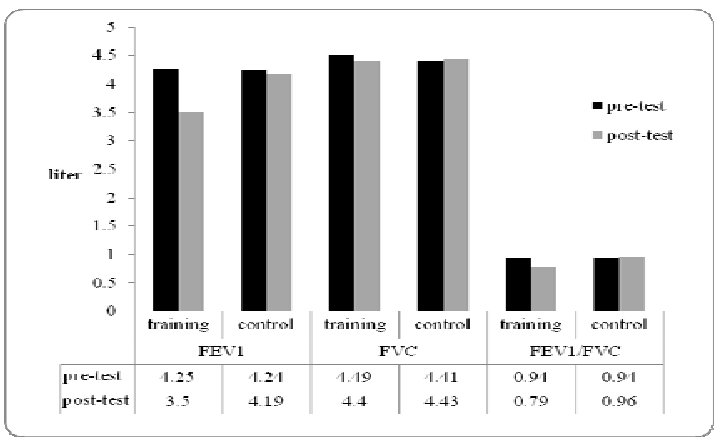

Figure 2

Comparison of pulmonary index under pre test and post test conditions of the two groups 


\section{Discussion}

The results showed that the difference between the exercise group and the control group in FEV1was statistically significant after the test protocol. On the other hand, one sub maximal incremental aerobic exercise session led to a significant decrease in the FEV1. Previous research indicated that three potential factors limit exercise capacity: diffusion capacity, ventilatory muscle power and limitation in expiration flow (Fatemi, 2009, Jackson, 1996).

In addition, it has been established that healthy individuals show an increase in ventilation associated with increased $\mathrm{VO}_{2}$ yet the respiratory system does not limit exercise capacity. Usually a $10-15 \%$ decrease in FEV1 signifies exercise-induced bronchospasm (Ehteshami, 2002, Rundell, 1999, Fatemi, 2009). However airway stimulus and inflammation induced exercise occurs due to the bronchospasm (Ucok, 2004, Smith, 2002, Mehmet, 2004). FEV1 is used as an index of airway obstruction. Obstructive pulmonary disease leads to a notable decrease in FEV1 and may be revealing less than $40 \%$ of vital capacity (VC) under this condition (Guyton, 2006). Thus in this study, the decrease in FEV1 is related to the exercise-induced bronchospasm.

However, some scientists reported that most likely, the reason for the significant decrease in these factors was fatigue of respiratory muscles (Ozturan, 1999). Thomase et al (2001) suggest that the increase in blood volume may be responsible for the decrease in pulmonary factors following exercise. Furthermore, this decrease is more in accordance with expiratory flow limitation rather than inspiratory limitation. Most likely this is due to an increase in residual volume after exercise.

Athletes with respiratory system limitations experience major impairment during exercise or competitions in hypoxic environments (Dempsey, 2008). This seems to be caused primarily by the excessive alveolar-to-arterial $\mathrm{O}_{2}$ difference $\left(\mathrm{A}-\mathrm{aDO}_{2}\right)$ and inadequate compensatory hyperventilation. It may also be a byproduct of the increasing temperature and metabolic acidosis which, in turn, causes a rightward shift in the $\mathrm{Hb}-\mathrm{O}_{2}$ dissociation curve (Dempsey, 1999). Helenius et al (2000) reported the prevalence rate of exercise-induced bronchospasm by $17 \%$ among endurance-runners and $8 \%$ in sprinters. Kohl et al (1997) holds that in functional respiratory changes after running competitions may have their roots in the fatigue of respiratory muscles, pulmonary obstruction, pulmonary edema, closing of the small airways and changes in pulmonary blood volume.

Our findings reveal that the FEV1/FVC was significantly decreased in post-test conditions in the experimental group. Likewise, we found a significant difference between the training and the control group in terms of the above-mentioned variable. The decrease in FEV1/FVC in the exercise group following the test might be contributed to the decrease in absolute volume of FEV1 and unchanged FVC. The FEV1/FVC factor varies and is related to the strength of expiratory muscles. A weakness of expiratory muscles leads to a quick fall in the expiratory ability. This lowers the FEV1/FVC factor even without the obstruction of airways. The FEV1/FVC factor is used as an indicator of response in the changes of inspiratory studies and exercise-induced spasm (Kohl, 1997).

Hiroaki et al (2003) showed that the decrease in a 6 -minute walk test at 1 and 2 weeks after COPD surgery is significantly correlated with the preoperative FEV1/FVC\% not VC.

Feinstein et al (1996) also studied the prevalence of asthma and exercise-induced bronchospasm in adult soccer players in a submaximal test and found that more than half of subjects showed EIA. As Feinstein (1996) concludes, many athletes can be identified as having abnormal pulmonary function by use of a submaximal step-test as an exercise protocol. Self-reporting questionnaires do not appear to be sensitive enough to identify athletes with this condition.

William and Terry (2002), in their study on pulmonary function, used different levels of intensity and duration of exercise and concluded that, rather than affecting FVC, 5 - 10 minutes after the exercise FEV1 decreases significantly. This decrease leads to a decline in FEV1/FVC ratio. Despite unequivocal opinions, it has been clear that the decrease in FEV1/FVC ratio indicates an increase in airway resistance and a decline in ventilation efficiency which can result in differences in endurance capacity of athletes (Mehmet, 2004, Peter, 2003, Voy, 1984).

Another interesting finding of this research was the nonexistence of any significant FVC difference between control and exercise group during the post test phase. William et al (2003) reported that physical training has no effect on FVC.

Furthermore, such a condition may result in differences in aerobic capacity for endurance athletes, which give rise to inherent advantages on these athletes' side (Ucok, 2004, Mehmet, 2004, Voy, 1984). Pulmonary functional adaptations and prolonged 
endurance training could be regarded as possible mechanisms which are associated with these conditions. Fred and colleagues (1976) studied the pulmonary volume changes with anti chemical masks at various resistances and maintained that the small added airway resistance commonly found in equipment for making different respiratory measurements during exercise can cause a decrement in endurance in proportion to the size of the resistance.

Likewise, type, intensity and duration of exercise, mental factors as fatigue, overtraining and arousal factors also can trigger EIA (Palatsi, 1980). The higher the intensity and duration of the exercise, the more severe of EIB (Jonathan, 2008). Besides, the diffusion capacity in athletes is higher than untrained individuals. The increase in lung volume might be regarded as one of the respective reasons (Olufeyi, 2002, Farid, 2005).

Although EIA is supposed to occur only during high intensity exercise (Dempsey, 2008, Mc Claran, 1998, Guenette, 2007), the present study indicates that submaximal exercise can also result in EIA. It seems necessary to mention that in addition to the duration and intensity of exercise, temperature and environmental pollutants can change absolute values of lungs and affect pulmonary functions (Alex, 2006, Jonathan, 2008, Farid, 2005). It suggests that more research is needed to be carried out pulmonary changes after exercise because these variables affected are by many other factors that need to be investigated in future studies.

\section{Conclusion}

Many athletes can be identified as having abnormal pulmonary function following submaximal exercise. These methods appear to be sensitive enough to identify athletes with this condition. Our results indicate that one submaximal incremental aerobic session of exercise causes a significant decrease in FEV1 and FEV1/FVC, and may be the cause of exercise-induced asthma.

\section{References}

Alex BG, Gordon JB. The effect of a competitive season and environmental factors on pulmonary function and aerobic power in varsity hockey players. Appl Physiol Nutr Metab, 2006. 31: 95-100.

Dempsey JA, Donald C, Mc Kenzie, Hans C, Haverkamp and Marlowe W, Eldridge. Update in the Understanding of Respiratory Limitations to Exercise Performance in Fit, Active Adults. CHEST 2008. 134:613-22

Dempsey JA, Wagner PD.Exercise-induced arterial hypoxemia. J Appl Physiol, 1999. 87: 1997-006.

Ehteshami- Afshar A, Asadian A, Zahmatkesh MM. Exercise - Induced Bronchospasm in Soccer Players. NRITLD, National Research Institute of Tuberculosis and Lung Disease, IranTanaffos 2002. 1(2), 35-39.

Farid R, Jabbari Azad F, Ebrahimi Atri A, Baradaran Rahimi M, Khaledan A, Talaei-Khoei M, et al. Effect of Aerobic Exercise Training on Pulmonary Function and Tolerance of Activity in Asthmatic Patients. Ir J Aller Asthma Immun 2005. 4(3):133-138.

Fatami R, Ghanbarzadeh M. Relationship between Airway Resistance indices and Maximal Oxygen Uptake in Young Adults. J Human Kinetics, 2009. 22: 29-34.

Feinstein RA, LaRussa J, Wang-Dohlman A, Bartolucci AA. Screening adolescent athletic for exercise-induced asthma. Clinical Journal of Sport Medicine, 1996. 6 (2):119-23.

Fred W, Stemler Francis N. Added Airway Resistance and Endurance in Intensive Exercise. Technical rept, 1976. P 31.

Guenette JA, Witt JD, McKenzie DC, Road JD, Sheel AW. Respiratory mechanics during exercise in endurancetrained men and women. J Physiol 2007. 581:1309-322.

Guyton A.C., Hall J.E,. Text book of Medical Physiology, 11th ed, Elsevier, Saunders and USA, 2006. P 526-529.

Helenius IJ, Haahtela T. Allergy and asthma in elite summer sport athletes. The Journal of Allergy and Clinical Immunology, 2000.106:444-52. 
Hiroaki N, Kenichi W,Takashi Ohtsuka Tsuguo N, Keiichi S. Six-minute walking and Pulmonary Function Test Outcomes during the Early Period after Lung Cancer Surgery with Special Reference to Patients with Chronic Obstructive Pulmonary Disease. Minato-ku, Tokyo 2003. 113- 19, Japan

Holger J, Schunemann Joan Dorn, Brydon JB, Grant, Warren Winkelstein, Jr., Trevisian M. Pulmonary function is the long term predictor of mortality in the general population: 29 years follow-up of the buffalo health study. Chest 2000. 118: 656-64.

Jackson AS, Wier LT, Ayers GW, Beard EF, Stuteville JE, Blair SN. Change in Aerobic power of woman ages 20-64 yr. Medicine science in sport and exercise, 1996. 28 (7), 884-91.

Jonathan PP., Christopher PB, Gary P David., Christopher K. Benjamin, et al. Airway Inflammation in ExerciseInduced Bronchospasm Occurring in Athletes without Asthma. Journal of Asthma, 2008. 45(5):363 - 67

Katz R.M., Exercise-induced asthma in the Olympic athlete. J Asthma 1992. 29(4): 227-8.

Kohl J, Koller EA, Brandenberger M, Cardenas M, Boutellier U. Effect of exercise-induced hyperventilation on airway resistance and cycling endurance. Eur J of Appl Physiol and Occup Physiol 1997. 75(4).

Mc Claran SR, Harms CA, Pegelow DF, Dempsey JA. Smaller lungs in women affect exercise hyperpnea. J Appl Physiol 1998. 84:1872-1881.

Medelli J, Lounana J, Messan F, Menuet J, Petitjean M. Testing of pulmonary function in a professional cycling team. Sports Medicine and physical Fitness, 2006. 46(2):298- 06.

Mehmet U, Turker S, Deniz N, Vakur A, Abidin K. The Prevalence of Exercise Induced Bronchoconstriction in elite athletics. Sports sciences and Med, 2004. 3(1): 57-59.

Nischom KS, Arthur TJ, William HS, Frack CK. Exercise-induced respiratory resistance changes as measured with the airflow perturbation device. Physiol Meas, 2005.26: 29-38.

Oguz O, Ramis O. Effects of Increased Respiratory Resistance on Maximal O2 Uptake and Anaerobic Threshold during Incremental Exercise Tests. Turk J Med Sci, 2002. 32: 49-52.

Olufeyi AA, Arogundade O. The effect of chronic exercise on lung function and basal metabolic rate in some Nigerian athlets. Afr J Biomed Res, 2002. 5: 9-11.

Ozturan D. Effect of acute on respiratory function tests of basketball players. Tip Dergisi, 1999. 21(1):10-14.

Palatsi IJ. Pulmonary function and maximal oxygen uptake in sprinters and endurance runners. Sports sciences, 1980. 2(2):59-62.

Peter HCK, Janjaap V, Jan LKimpen, Paul JM, Cornelis K. Longitudinal Determinants of Peak Aerobic Performance in Children with Cystic Fibrosis. CHEST, 2003. 124:2215-219.

Prajapati R, Upadhyay Dhungel K, Pramanik T, Ghosh A, Roychowdhury P. Assessment of some pulmonary parameters and cardiorespiratoryfitness status in Nepalese medical students. Nepal Med Coll J. 2008.10(1): 2829.

Prakash S, Mesh Ram S, Ramtekkar U. Athletes, yogis and individual with sedentary life styles; do their lung function differ?. Indian J Physiol Pharmacol, 2007.51: 76-80.

Provost-Craig MA, Arbour KS, Sestili DC. The incidence of exercise-induced bronchospasm in competitive figure skaters. J Asthma, 1996.33(1): 67-71.

Rundell KW, Jenkison DM. Exercise-Induced Bronchospasm in elite athletes. Sports Med, 1999.32, 583-600.

Smith E, Mahony N, Donne B, Brien MO. Prevalence of obstructive in Irish collegiate athletic. Irish Journal of Medical Science, 2002.171(4):202- 05.

Tan RA, Spector SL. Exercise-induced asthma. Sports Med 1998; 25(1): 1-6 
Thomas J, Wetter CM, Croix DF, Pegelow DA, Sonetti, Jerome AD. Effects of exhaustive endurance exercise on pulmonary gas exchange and airway function in women. Appl Physiol, 2001. 91: 847-58.

Ucok K, Dane S, Gokbe H, Akar S. Prevalence of Exercise-Induced Bronchospasm in long distance runners trained in cold weather. Medicine and science in sports and Exercise, 2004.182(5):265- 70

Voy RO. The Olympic Committee experience with Exercise-Induced Bronchospasm. Medical Science and sports exercise, 1984.18:328-30.

William BK, Marian M, Andrew M, Michael WP, Rosemary GH, Lorilie AW et al. Pulmonary limits to submaximal exercise in reheumatoid arthritis. An International Electronic Journal, 2003. 6(2).

William EA, Terry LD. The effects of respiratory muscles training on Vo2max, the ventilaory threshold and pulmonary function. J Exerc Physiol, 2002. 5 (2):29-35.

\section{Acknowlegdement}

This study was carried out in the Department of Sport Physiology, faculty of Physical Education and Sport Sciences, Shahid Chamran University of Ahvaz, Iran. The authors would like to sincerely thank all participants in this study and the staff of Physical Education and Sport Sciences faculty.

\section{Corresponding author}

\section{Rouholah Fatemi}

Faculty of Physical Education \& Sports Science

Shahid Chamran University- Iran

phone: +98 0611-3367008 - +989171484369

fax: +98 0611-3332618

e-mail: r.fatemi61@gmail.com 